\title{
THE PHYSICAL MEANING OF THE TITIUS-BODE FORMULA
}

\author{
V.A.Smirnov \\ National Academy of Communications, Odessa, Ukraine \\ smirnovw@tm.odessa.ua
}

ABSTRACT. It is proposed an special hypothesis of the formation of symmetric objects in space with the help of the interference of standing waves which act in the process of evolution of the primordial gas-dust cloud including the formation of the Solar system, that may be revealed by 11-year cycle of Solar activity in particular.

It is used the idea of J.Kepler of the construction of the Solar system, viewed as inscribed and circumscribed Platonic figures, a common measure of which, according to the author, is the length of the standing waves generated by the variable energy sources of the primary cloud. This explains the physical meaning of the Titius-Bode formula.

In 1968 S.F.Dermott revealed the foundation of TitiusBode empiric formula, basing on Theory of Resonance. But in addition to direct statement of existing resonance mechanism of formula $\mathrm{T}(\mathrm{n})=\mathrm{T}(0) \cdot \mathrm{C}^{\mathrm{n}}, \mathrm{n}=1,2,3,4$, physical sense does not come to light. I tried to explain the formation of symmetric figures and space constructions with evident experience of formation of Chladni E. figures, offering the calculation, confirmed by experience of appearance of new space bodies in the nodes of standing waves of primary gas-dust cloud. The interference of standings waves is surely promotes the formation of different figures - such as symmetric galaxies, hexagon vortex at Saturn, and others. Unlike Dermott's theory, our explanation of symmetric formation of planets is a common solution of task for multi bodies interaction, and proposes the version of solution in case with more than two bodies.

Key words: Standing Waves, Chladni Experiments, Nodes

The process of evolution of the solar system means the development of the structure of gas-dust cloud after the initial impulse by way of the impact in most cases of a supernova explosion. Thus the wave motions are practically excluded from consideration. As the experience shows at the time of the formation of standing waves with the observed acoustic resonance the wave motions at the nodal points can accumulate clumps of matter that make up the primary cloud. A similar pattern is observed in the experiments of Chladni E. (Smirnov, 1999).

J.Kepler's plan of the solar system, which took into account the distribution of the planets according to their distance to the Sun, was built as a series of inscribed and circumscribed Platonic figures (Kepler, 1939). According to his scheme the average distances of the planets to the Sun could be obtained in the form of the radiuses of the circumscribed spheres (Fig. 1). This fact indicates the existence of a common measure of the Platonic figures constructed in such a way.

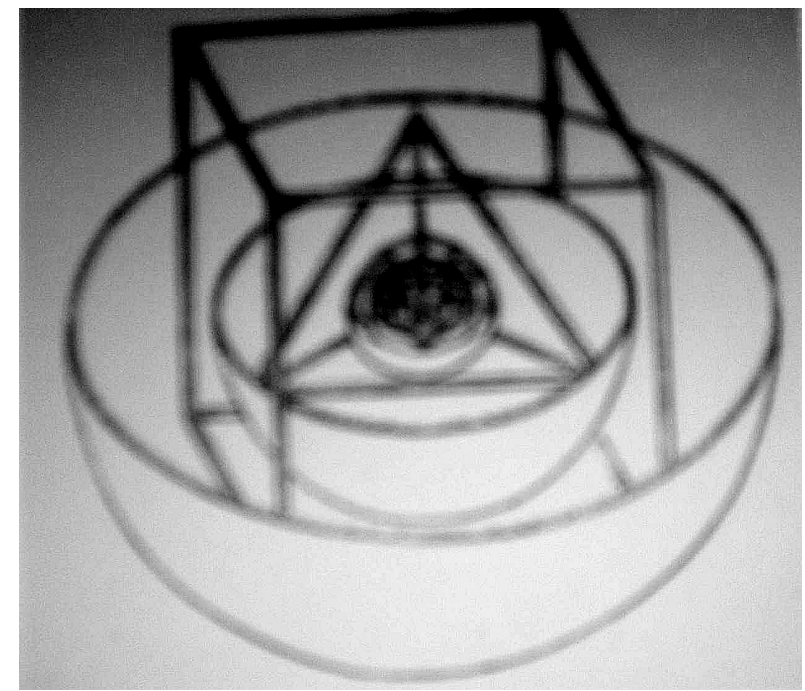

Figure 1: In the work of Johannes Kepler "Welt-Harmonik" the great scientist builds a plan of a distribution of planets in the solar system in the form of inscribed and described Platonic figures (see page 287). Using since the time of Huygens the well - known concept of wavelength and frequency fluctuations in the standing wave one can build a plan of the solar system taking into account these concepts which duplicate dimensions in the formula Titius - Bode.

In the time of Kepler the concepts of the wavelength were not yet used. That's why Kepler could come to the conclusion that the length of a standing wave $\lambda$, emitted by the central formation of the Solar system that forms waves of energy into space, which are shaping with the reflected waves from the interface of more dense environmental conditions of the gaseous nebula and less dense environmental conditions of the surrounding space, could serve as a common measure for measuring distances of the planets to the Sun. If the standing wave in the onedimensional case of the reflection from a less dense medium is formed in the $\mathrm{Y}$ axis direction with the displacement $\mathrm{X}$, the wave equation can be written as:

$$
X=a \cos \frac{2 \pi}{\lambda} Y \cos \frac{2 \pi}{T} t
$$

The planets are being formed in the nodes generated in the wave where the oscillation amplitude is zero. In astronomical units the distances to the Sun are determined at the points along the axis $Y=\frac{2 n+1}{4} \cdot \lambda$, wherein $\mathrm{n}=0,1,2 \ldots$ 
The comparison of the observed and calculated distances to the planets to the Sun and the distances of the satellites to the planets according to the proposed wave principle one can find in the author's work (Smirnov, 2001).

Using this technique, we present the tables of calculated and measured distances of the planets to the Sun, Jupiter's satellites to the central planets and satellites of Saturn and Uranus to the central planets. Taking a common measure of the Platonic figures or the length of the standing wave $\lambda$, which forms the Solar system, $\lambda=0,52 \mathrm{AU}$, and taking an active place of the primary Sun which forms standing waves as the first term in the calculation formulas that determine the distances of the planets to the Sun through the position of the nodes of standing waves in the primary gas-dust cloud, we obtain table 1 .

Table 1: Distances of the planets to the Sun expressed across the nodes of standing wave $\lambda$

\begin{tabular}{|l|c|c|}
\hline Planet & $\begin{array}{c}\text { True distance } \\
\text { the planets } \\
\text { to the Sun } \\
\text { (AU) }\end{array}$ & $\begin{array}{c}\text { Calculated the } \\
\text { distance of } \\
\text { planet to the Sun } \\
\text { (AU) }\end{array}$ \\
\hline Mercury & 0,39 & 0,72 \\
\hline Venus & 1,00 & $0,39+1,50 \lambda=1,17$ \\
\hline Earth & 1,52 & $1,00+1,00 \lambda=1,52$ \\
\hline Mars & 2,90 & $1,00+4,00 \lambda=3,08$ \\
\hline Main asteroid belt & 5,20 & $1,00+8,00 \lambda=5,16$ \\
\hline Jupiter & 9,54 & $1,00+16,0 \lambda=9,32$ \\
\hline Saturn & 19,20 & $1,00+35,0 \lambda=19,20$ \\
\hline Uranus & 30,10 & $1,00+56,0 \lambda=30,12$ \\
\hline Neptune & 39,50 & $1,00+74,0 \lambda=39,48$ \\
\hline Pluto & &
\end{tabular}

Taking into account the length of the standing wave to be $\lambda=240.10^{3} \mathrm{~km}$ for the system of satellites of Jupiter, we can derive the result similarly, as shown below:

Table 2.

\begin{tabular}{|l|c|l|}
\hline $\begin{array}{c}\text { Satellites of } \\
\text { Jupiter }\end{array}$ & $\begin{array}{c}\text { True distance to } \\
\text { Jupiter }\left(10^{3} \mathrm{~km}\right)\end{array}$ & $\begin{array}{c}\text { Calculated distance } \\
\text { to Jupiter }\left(10^{3} \mathrm{~km}\right)\end{array}$ \\
\hline 5 & 181 & \\
\hline Io & 422 & $181+\lambda=422$ \\
\hline Europa & 671 & $181+2 \lambda=661$ \\
\hline Ganymede & 1070 & $181+4 \lambda=1141$ \\
\hline Callisto & 1883 & $181+7 \lambda=1861$ \\
\hline 6 & 11476 & $181+47 \lambda=11461$ \\
\hline 10 & 11700 & $181+48 \lambda=11701$ \\
\hline 7 & 11737 & $181+48 \lambda=11701$ \\
\hline 12 & 21200 & $181+88 \lambda=21301$ \\
\hline 11 & 22600 & $181+93 \lambda=22501$ \\
\hline 8 & 23500 & $181+97 \lambda=23461$ \\
\hline 9 & 23600 & $181+98 \lambda=23701$ \\
\hline
\end{tabular}

From the Table of Jupiter satellites we notice that the $6^{\text {th }}, 10^{\text {th }}$ and $7^{\text {th }}$ satellite, apparently, formed in the same node of the standing wave creating them. The same thing can be noticed concerning the $8^{\text {th }}$ and $9^{\text {th }}$ satellite. Between Calisto and the $6^{\text {th }}$ satellite, probably, unnoticed masses of substances exist.

If we take as the "common measure" the length of characteristic standing wave for Saturn, $\lambda=27.10^{3} \mathrm{~km}$, then the distances of the Saturnian system of satellites displays the same wave regularity (Table 3 ).

If we take $\lambda=62.10^{3} \mathrm{~km}$ as the effective wave length for the system of Uranian satellites were formed (Table 4).

Table 3.

\begin{tabular}{|l|c|l|}
\hline $\begin{array}{c}\text { Saturnian } \\
\text { Satellite }\end{array}$ & $\begin{array}{c}\text { True distance to } \\
\text { Saturn }\left(10^{3} \mathrm{~km}\right)\end{array}$ & $\begin{array}{c}\text { Calculated distance } \\
\text { to Saturn }\left(10^{3} \mathrm{~km}\right)\end{array}$ \\
\hline Janus & 159 & $159+\lambda=186$ \\
\hline Mimas & 186 & $159+3 \lambda=240$ \\
\hline Enceladus & 238 & $159+5 \lambda=294$ \\
\hline Tethys & 295 & $159+8 \lambda=375$ \\
\hline Dione & 377 & $159+14 \lambda=537$ \\
\hline Rhea & 527 & $159+39 \lambda=1212$ \\
\hline Titan & 1222 & $159+49 \lambda=1482$ \\
\hline Hyperion & 1483 & $159+126 \lambda=3561$ \\
\hline Iapetus & 3560 & $159+474 \lambda=12957$ \\
\hline Phoebe & 12950 &
\end{tabular}

The error in most cases one sees almost complete coincidence of the calculated and experimental distances of planets to the Sun. As can be seen, in the basis of existence lies the universal wave principle and the spacetime frames of the "golden section".

Table 4.

\begin{tabular}{|l|c|c|}
\hline $\begin{array}{c}\text { Uranian } \\
\text { Satellite }\end{array}$ & $\begin{array}{c}\text { True distance } \\
\text { to Uranus } \\
\left(10^{3} \mathrm{~km}\right)\end{array}$ & $\begin{array}{c}\text { Calculated } \\
\text { distance to Uranus } \\
\left(10^{3} \mathrm{~km}\right)\end{array}$ \\
\hline Miranda & 130 & $130+\lambda=192$ \\
\hline Ariel & 192 & $130+2 \lambda=254$ \\
\hline Umbriel & 267 & $130+5 \lambda=440$ \\
\hline Titania & 438 & $130+7 \lambda=564$ \\
\hline Oberon & 586 & \\
\hline
\end{tabular}




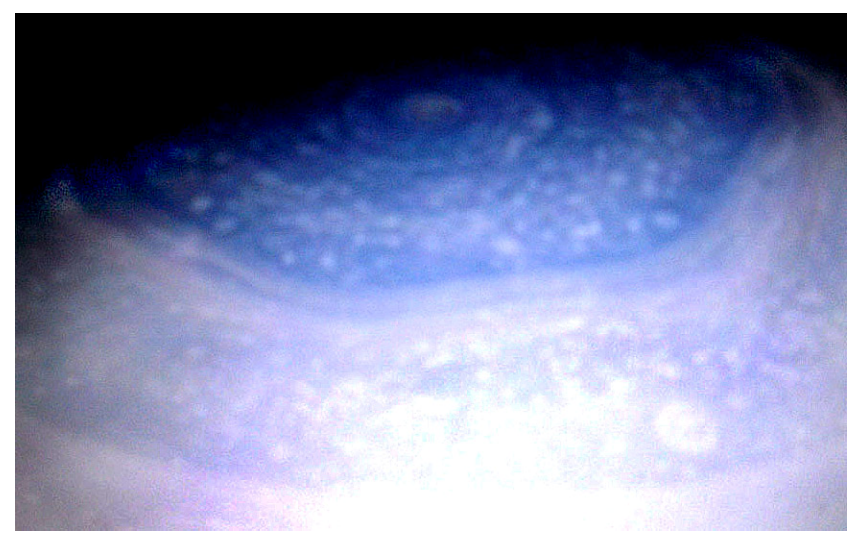

Figure 2: The whirlwind of the regular hexagonal structure in the northern hemisphere of Saturn, photographed by Cassini mission November 28, 2013 at a distance of $610.4 \mathrm{~km}$. Formation diameter is $25,000 \mathrm{~km}$.

The above formula for the distances of the planets to the Sun and the distances of the planets to their satellites, reveals the physical meaning of the well-known formula, composed empirically by Bode - Titius:

$$
Y=0,4+0,3 \cdot 2^{\mathrm{n}}, \text { wherein } \mathrm{n}=-\infty, 1,2,4,5 \ldots
$$

Note that in some cases the standing waves are responsible for the formation of symmetrical shapes of galaxies by cosmic objects that resemble the inscribed and circumscribed Platonic figures and the vortex formation in the form of hexagon on Saturn recently shown on the Internet.

According to the observations, the elementary calculation shows that the hexagon vortex is formed by a standing wave with a wavelength $\lambda=12500 \mathrm{~km}$ (Fig. 2).

According to the reports of the Hubble telescope's observations in outer space, the energy waves are observed in the substance of the outer space while the evolution of galaxies and other objects, and the length of these energy waves reaches $\lambda$ hundreds of light years.

Generally it is obvious that the discovery made with the help of the Hubble telescope of symmetrically constructed shapes of galaxies which echo the view of the Kepler-

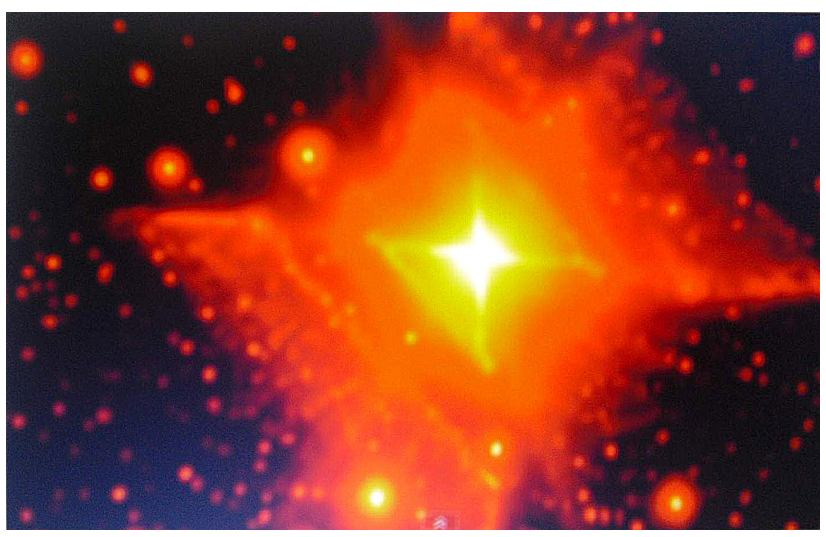

Figure 3: The Nebula MWC 922, formed in the shape of symmetrically arranged figures during the interference of standing waves.

figures showing the plan of the solar system can be explained only through the formation of the giant-scale energetic standing waves. For only in such cases of the formation of standing waves the distances between nodes and antinodes positions remain unchanged in time. This fact can explain the shape of the nebula MWC 922 (Fig. 3) Similarly, the appearance in the walls of the fixed giant vortex on Saturn as a hexagon explains the formation of its standing wave in the wall. Another explanation of these phenomena can hardly be found.

\section{References}

Dermott S.F.: 1968, Mon. Not. RAS, 141, 363.

Dermott S.F.: 1969, Mon. Not. RAS, 142, 143.

Kepler J., Welt-Harmonik, Verlag R.Oldenbourg, Munchen-Berlin, 1939, 403 p.

Smirnov V.A.: 1999, Astronomical and Astrophysical Transaction, 18, 521.

Smirnov V.A.: 2001, in: Proc of the International Meteor Conf., Cerkno, Slovenia, 20-23 September 2001, 64. 